Research Article

\title{
Cosine Similarity Measure between Hybrid Intuitionistic Fuzzy Sets and Its Application in Medical Diagnosis
}

\author{
Donghai Liu (D), ${ }^{1}$ Xiaohong Chen, ${ }^{2}$ and Dan Peng ${ }^{1}$ \\ ${ }^{1}$ Department of Mathematics, Hunan University of Science and Technology, Xiangtan, China \\ ${ }^{2}$ Hunan University of Commerce, Changsha, China \\ Correspondence should be addressed to Donghai Liu; donghailiu@126.com
}

Received 24 January 2018; Revised 11 June 2018; Accepted 12 September 2018; Published 17 October 2018

Guest Editor: Miguel García-Torres

Copyright (c) 2018 Donghai Liu et al. This is an open access article distributed under the Creative Commons Attribution License, which permits unrestricted use, distribution, and reproduction in any medium, provided the original work is properly cited.

In this paper, a cosine similarity measure between hybrid intuitionistic fuzzy sets is proposed. The aim of the paper is to investigate the cosine similarity measure with hybrid intuitionistic fuzzy information and apply it to medical diagnosis. Firstly, we construct the cosine similarity measure between hybrid intuitionistic fuzzy sets, and the relevant properties are also discussed. In order to obtain a reasonable evaluation in group decision, the weight of experts under different attributes is determined by the projection of individual decision information on the ideal decision information, where the ideal decision information is the average values of each expert's evaluation. Furthermore, we propose a decision method for medical diagnosis based on the cosine similarity measure between hybrid intuitionistic fuzzy sets, and the patient can be diagnosed with the disease according to the values of proposed cosine similarity measure. Finally, an example is given to illustrate feasibility and effectiveness of the proposed cosine similarity measure, which is also compared with the existing similarity measures.

\section{Introduction}

A similarity measure is an important tool for determining the degree of similarity between two objects in many fields, such as pattern recognition, medical diagnosis, and so on. Many similarity measures have been introduced [1-8]. Among them, some similarity measures of intuitionistic fuzzy sets (IFSs) have been proposed. For example, Li and Cheng [3] proposed a similarity measure between IFSs and applied it to pattern recognition. Huang and Yang [2] defined the similarity measure between IFSs based on the Hausdorff distance and used it to calculate the degree of similarity between IFSs. Nguuen [9] proposed a new knowledge-based similarity measure between IFSs and applied it to pattern recognition. However, due to the complexity and uncertainty of the decision-making environment, the membership degree and nonmembership degree of IFS need to be expressed by interval rather than the numerical value. Motivated by this, Atanassov and Gargov [10] introduced the concept of interval-valued intuitionistic fuzzy set (IVIFS), which is a generalization of IFS. $\mathrm{Xu}$ [11] proposed some distance and similarity measures between IVIFSs and applied them to pattern recognition.
On the other hand, the cosine similarity measure based on Bhattacharyya distance was first proposed in Bhattacharyya [12]. Ye [7] proposed a cosine similarity measure for IFSs $\left(C_{\mathrm{IFS}}\right)$ and applied it to pattern recognition. Furthermore, Ye [13] proposed the cosine similarity measure for IVIFSs $\left(C_{\text {IVIFS }}\right.$ ) and applied it to group decision-making problems. However, in the complex group decision-making problem, it is difficult to use a single value to express the alternative under all attributes. Because some attributes might be represented by IFSs, but other attributes are suitable to be represented by IVIFSs. At this time, the people should use hybrid intuitionistic fuzzy set to make a decision. However, the existing methods can not deal with the hybrid fuzzy information. As far as we know, no people studied the cosine similarity measure between hybrid IFSs. Motivated by this, we will introduce the cosine similarity measure with hybrid intuitionistic fuzzy information $\left(C_{\mathrm{HIFS}}\right)$ in this paper. This generalization makes the $C_{\mathrm{HIFS}}$ measure includes $C_{\mathrm{IFS}}$ measure and $C_{\text {IVIFS }}$ measure as particular case.

In addition, applying the $C_{\mathrm{HIFS}}$ measure to group decision-making problems is very interesting. For example, Zhou and Wahab [14] use transmissibility incorporated 
with cosine similarity measure to investigate the structural damage detection. Furthermore, Zhou et al. [15] apply transmissibility function with distance measure to separate the intact patterns apart from the damaged pattern. In group decision-making problems, the weight of the experts under different attributes can be obtained by using the projection of individual decision information on the ideal decision information. Then, we aggregate all individual decisions into a collective one and apply the proposed cosine similarity measure between hybrid intuitionistic fuzzy sets to medical diagnosis.

The rest of the paper is organized as follows. In Section 2 , we review the cosine similarity measure for IFSs and IVIFSs. In Section 3, we propose the $C_{\text {HIFS }}$ measure, some properties are also analyzed. In Section 4, we propose a decision method for medical diagnosis based on the cosine similarity measure between hybrid intuitionistic fuzzy sets. In Section 5, an example is given to illustrate the feasibility and effectiveness of the proposed $C_{\mathrm{HIFS}}$ measure. Finally, the conclusion and further research are discussed in Section 6.

\section{Preliminaries}

Throughout this paper, let $X=\left\{x_{1}, x_{2}, \ldots, x_{n}\right\}$ be a finite universal set. In this section, we briefly review the IFSs and IVIFSs, the cosine similarity measure between IFSs, and the cosine similarity measure between IVIFSs.

\subsection{Intuitionistic Fuzzy Set}

Definition 1. Let $X$ be a fixed set, an intuitionistic fuzzy set (IFS) $A$ in $X$ is defined as:

$$
A=\left\{\left(x_{j}, \mu_{A}\left(x_{j}\right), v_{A}\left(x_{j}\right) \mid x_{j} \in X\right)\right\},
$$

where the functions $\mu_{A}\left(x_{j}\right)$ and $v_{A}\left(x_{j}\right)$ represent the membership degree and nonmembership degree of the element $x_{j}$ to the set $A$, respectively, such that $0 \leq$ $\mu_{A}\left(x_{j}\right)+v_{A}\left(x_{j}\right) \leq 1 \forall x_{j} \in X$.

The intuitionistic fuzzy index $\pi_{A}\left(x_{j}\right)=1-\mu_{A}\left(x_{j}\right)-$ $v_{A}\left(x_{j}\right)$, and we have $0 \leq \pi_{A}\left(x_{j}\right) \leq 1$. For example, $A=$ $(0.4,0.3)$ is an intuitionistic fuzzy number, and $\pi_{A}=0.3$. The space of membership degree of IFS is shown in Figure 1.

In particular, when $X$ has only one element, the IFS $A=$ $\left\{\left(x_{j}, \mu_{A}\left(x_{j}\right), v_{A}\left(x_{j}\right) \mid x_{j} \in X\right)\right\}$ is reduced to $A=\left(\mu_{A}\left(x_{j}\right)\right.$, $\left.v_{A}\left(x_{j}\right)\right)$, which we call it an intuitionistic fuzzy number (IFN).

For any two IFSs $A=\left(x_{j}, \mu_{A}\left(x_{j}\right), v_{A}\left(x_{j}\right)\right)$ and $B=$ $\left(x_{j}, \mu_{B}\left(x_{j}\right), v_{B}\left(x_{j}\right)\right)$, the following operations are true [16]:

(1) $A+B=\left(\mu_{A}\left(x_{j}\right)+\mu_{B}\left(x_{j}\right)-\mu_{A}\left(x_{j}\right) \mu_{B}\left(x_{j}\right), v_{A}\left(x_{j}\right)\right.$ $\left.v_{B}\left(x_{j}\right)\right)$

(2) $\lambda A=\left(1-\left(1-\mu_{A}\left(x_{j}\right)\right)^{\lambda},\left(\nu_{A}\left(x_{j}\right)\right)^{\lambda}\right), \lambda>0$

(3) $A=B$ if $\mu_{A}\left(x_{j}\right)=\mu_{B}\left(x_{j}\right), v_{A}\left(x_{j}\right)=v_{B}\left(x_{j}\right)$

The results of the operations $A+B$ and $\lambda A$ are still IFSs.

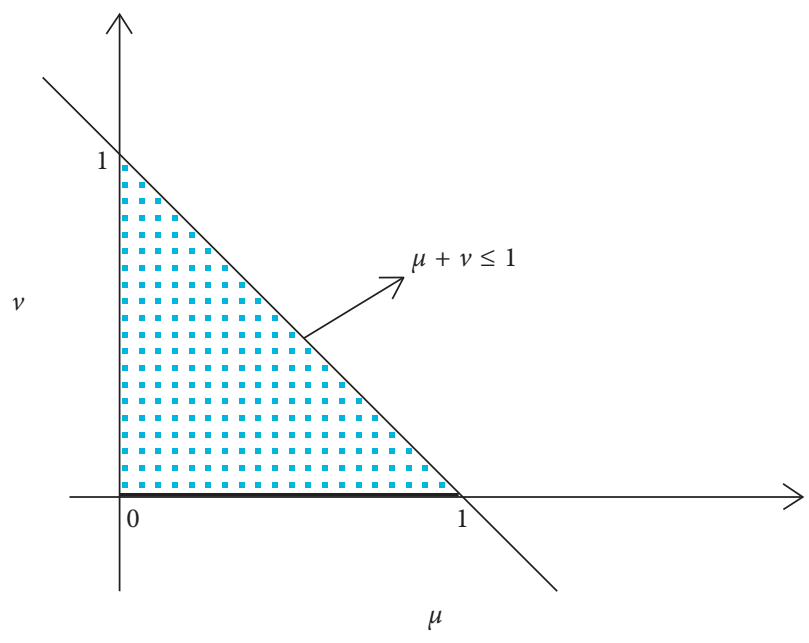

Figure 1: The membership degree of IFS.

\subsection{Interval-Valued Intuitionistic Fuzzy Set}

Definition 2. Let $X$ be a fixed set, an interval-valued intuitionistic fuzzy set $\widetilde{A}$ is defined as follows:

$\widetilde{A}=\left\{\left(x_{j},\left[\mu_{\widetilde{A} L}\left(x_{j}\right), \mu_{\widetilde{A} U}\left(x_{j}\right)\right],\left[\nu_{\widetilde{A} L}\left(x_{j}\right), \nu_{\widetilde{A} U}\left(x_{j}\right)\right]\right) \mid x_{j} \in X\right\}$,

where

$\mu_{\widetilde{A} L}\left(x_{j}\right) \geq 0, v_{\widetilde{A} L}\left(x_{j}\right) \geq 0 ; 0 \leq \mu_{\widetilde{A} U}\left(x_{j}\right)+v_{\widetilde{A} U}\left(x_{j}\right) \leq 1, \quad \forall x_{j} \in X$.

The interval-valued intuitionistic fuzzy index is defined as $\pi_{\widetilde{A}}\left(x_{j}\right)=\left[\pi_{\widetilde{A} L}\left(x_{j}\right), \pi_{\widetilde{A} U}\left(x_{j}\right)\right]$, where

$$
\begin{aligned}
\pi_{\widetilde{A} L}\left(x_{j}\right) & =1-\mu_{\widetilde{A} U}\left(x_{j}\right)-\nu_{\widetilde{A} U}\left(x_{j}\right), \pi_{\widetilde{A} U}\left(x_{j}\right) \\
& =1-\mu_{\widetilde{A} L}\left(x_{j}\right)-v_{\widetilde{A} L}\left(x_{j}\right) .
\end{aligned}
$$

For example, $\widetilde{A}=([0.3,0.4],[0.1,0.4])$ is an intervalvalued intuitionistic fuzzy number, the fuzzy index $\pi_{\widetilde{A}}\left(x_{j}\right)=[0.2,0.6]$.

Remark 1. If $\mu_{\widetilde{A} L}\left(x_{j}\right)=\mu_{\widetilde{A} U}\left(x_{j}\right), \nu_{\widetilde{A} L}\left(x_{j}\right)=v_{\widetilde{A} U}\left(x_{j}\right)$, then the interval-valued intuitionistic fuzzy set is reduced to intuitionistic fuzzy set.

When the set $X$ has only one element, the IVIFS $\widetilde{A}=$ $\left\{\left(x_{j},\left[\mu_{\widetilde{A} L}\left(x_{j}\right), \mu_{\widetilde{A} U}\left(x_{j}\right)\right],\left[\nu_{\widetilde{A} L}\left(x_{j}\right), \nu_{\widetilde{A} U}\left(x_{j}\right)\right]\right) \mid x_{j} \in X\right\}$ is reduced to $\widetilde{A}=\left(\left[\mu_{\widetilde{A} L}\left(x_{j}\right), \mu_{\widetilde{A} U}\left(x_{j}\right)\right],\left[\nu_{\widetilde{A} L}\left(x_{j}\right), \nu_{\widetilde{A} U}\left(x_{j}\right)\right]\right)$, which we call it an interval-valued intuitionistic fuzzy number(IVIFN).

Let $\quad \widetilde{A}=\left(x_{j},\left[\mu_{\widetilde{A} L}\left(x_{j}\right), \mu_{\widetilde{A} U}\left(x_{j}\right)\right],\left[\nu_{\widetilde{A} L}\left(x_{j}\right), \nu_{\widetilde{A} U}\left(x_{j}\right)\right]\right)$ and $\widetilde{B}=\left(x_{j},\left[\mu_{\widetilde{B} L}\left(x_{j}\right), \mu_{\widetilde{B} U}\left(x_{j}\right)\right],\left[\nu_{\widetilde{B} L}\left(x_{j}\right), \nu_{\widetilde{B} U}\left(x_{j}\right)\right]\right)$ be two IVIFSs, the following operations are true [17]:

(1) $\widetilde{A}+\widetilde{B}=\left(\left[\mu_{\widetilde{A} L}\left(x_{j}\right)+\mu_{\widetilde{B} L}\left(x_{j}\right)-\mu_{\widetilde{A} L}\left(x_{j}\right) \mu_{\widetilde{B} L}\left(x_{j}\right), \mu_{\widetilde{A} U}\right.\right.$ $\left.\left(x_{j}\right)+\mu_{\widetilde{B} U}\left(x_{j}\right)-\mu_{\widetilde{A} U}\left(x_{j}\right) \mu_{\widetilde{B} U}\left(x_{j}\right)\right],\left(\left[\nu_{\widetilde{A} L}\left(x_{j}\right) \nu_{\widetilde{B} L}\left(x_{j}\right)\right.\right.$, $\left.\left.\nu_{\widetilde{A} U}\left(x_{j}\right) \nu_{\widetilde{B} U}\left(x_{j}\right)\right]\right)$

(2) $\lambda \widetilde{A}=\left(\left[1-\left(1-\mu_{\widetilde{A} L}\left(x_{j}\right)\right)^{\lambda}, 1-\left(1-\mu_{\widetilde{A} U}\left(x_{j}\right)\right)^{\lambda}\right],\left[\left(\nu_{\widetilde{A} L}\right.\right.\right.$ $\left.\left.\left.\left(x_{j}\right)\right)^{\lambda},\left(\nu_{\widetilde{A} U}\left(x_{j}\right)\right)^{\lambda}\right]\right), \lambda>0$ 
(3) $\widetilde{A}=\widetilde{B} \quad$ if $\quad \mu_{\widetilde{A} L}\left(x_{j}\right)=\mu_{\widetilde{B} L}\left(x_{j}\right), \mu_{\widetilde{A} U}\left(x_{j}\right)=\mu_{\widetilde{B} U}\left(x_{j}\right)$, $\nu_{\widetilde{A} L}\left(x_{j}\right)=v_{\widetilde{B} L}\left(x_{j}\right)$ and $\nu_{\widetilde{A} U}\left(x_{j}\right)=v_{\widetilde{B} U}\left(x_{j}\right)$

\subsection{Cosine Similarity Measures for IFSs or IVIFSs.}

Definition 3 (Ye [7]). Let $A=\left(\mu_{A}\left(x_{j}\right), v_{A}\left(x_{j}\right)\right)$ and $B=\left(\mu_{B}\left(x_{j}\right), v_{B}\left(x_{j}\right)\right)$ be two IFSs in $X$, the cosine similarity measure between $A$ and $B$ is defined as follows:

$$
C_{\mathrm{IFS}}(A, B)=\frac{1}{n} \sum_{i=1}^{n} \frac{\mu_{A}\left(x_{i}\right) \mu_{B}\left(x_{i}\right)+v_{A}\left(x_{i}\right) \nu_{B}\left(x_{i}\right)}{\sqrt{\mu_{A}^{2}\left(x_{i}\right)+v_{A}^{2}\left(x_{i}\right)} \sqrt{\mu_{B}^{2}\left(x_{i}\right)+v_{B}^{2}\left(x_{i}\right)}} .
$$

The cosine similarity measure between two IFSs $A$ and $B$ satisfies the following properties:

(1) $0 \leq C_{\mathrm{IFS}}(A, B) \leq 1$

(2) $C_{\mathrm{IFS}}(A, B)=C_{\mathrm{IFS}}(B, A)$

(3) $C_{\text {IFS }}(A, B)=1$ if $A=B$

Definition 4 (Ye [13]). Let $\widetilde{A}=\left(\left[\mu_{\widetilde{A} L}\left(x_{j}\right), \mu_{\widetilde{A} U}\left(x_{j}\right)\right],\left[v_{\widetilde{A} L}\right.\right.$ $\left.\left.\left(x_{j}\right), \nu_{\widetilde{A} U}\left(x_{j}\right)\right]\right)$ and $\widetilde{B}=\left(\left[\mu_{\widetilde{B} L}\left(x_{j}\right), \mu_{\widetilde{B} U}\left(x_{j}\right)\right]\right),\left[\nu_{\widetilde{B C}}\left(x_{j}\right), \nu_{\widetilde{B U}}\right.$ $\left(x_{j}\right)$ ] be two IVIFSs in $X$, the cosine similarity measure between two IVIFSs $\widetilde{A}$ and $\widetilde{B}$ is defined as follows:

$$
C_{\text {IVIFS }}(\widetilde{A}, \widetilde{B})=\frac{1}{n} \sum_{i=1}^{n} \frac{\mu_{\widetilde{A} L}\left(x_{i}\right) \mu_{\widetilde{B} L}\left(x_{i}\right)+\mu_{\widetilde{A} U}\left(x_{i}\right) \mu_{\widetilde{B} U}\left(x_{i}\right)+v_{\widetilde{A} L}\left(x_{i}\right) v_{\widetilde{B} L}\left(x_{i}\right)+v_{\widetilde{A} U}\left(x_{i}\right) v_{\widetilde{B} U}\left(x_{i}\right)+\pi_{\widetilde{A} L}\left(x_{i}\right) \pi_{\widetilde{B} L}\left(x_{i}\right)+\pi_{\widetilde{A} U}\left(x_{i}\right) \pi_{\widetilde{B} U}\left(x_{i}\right)}{\sqrt{\mu_{\widetilde{A} L}^{2}\left(x_{i}\right)+\mu_{\widetilde{A} U}^{2}\left(x_{i}\right)+v_{\widetilde{A} L}^{2}\left(x_{i}\right)+v_{\widetilde{A} U}^{2}\left(x_{i}\right)+\pi_{\widetilde{A} L}^{2}\left(x_{i}\right)+\pi_{\widetilde{A} U}^{2}\left(x_{i}\right)} \cdot|H|},
$$

where

$$
|H|=\sqrt{\mu_{\overparen{B} L}^{2}\left(x_{i}\right)+\mu_{\overparen{B} U}^{2}\left(x_{i}\right)+\nu_{\overparen{B} L}^{2}\left(x_{i}\right)+\nu_{\overparen{B} U}^{2}\left(x_{i}\right)+\pi_{\overparen{B} L}^{2}\left(x_{i}\right)+\pi_{\overparen{B} U}^{2}\left(x_{i}\right)} .
$$

The cosine similarity measure between two IVIFs $\widetilde{A}$ and $\widetilde{B}$ satisfies the following properties:

(1) $0 \leq C_{\text {IVIFS }}(\widetilde{A}, \widetilde{B}) \leq 1$

(2) $C_{\text {IVIFS }}(\widetilde{A}, \widetilde{B})=C_{\text {IVIFS }}(\widetilde{B}, \widetilde{A})$

(3) $C_{\text {IVIFS }}(\widetilde{A}, \widetilde{B})=1$ if $\widetilde{A}=\widetilde{B}$

\section{Cosine Similarity Measure with Hybrid Intuitionistic Fuzzy Information}

In this section, we will propose the cosine similarity measure with hybrid intuitionistic fuzzy information $\left(C_{\mathrm{HIFS}}\right)$ and some properties are also discussed.

Definition 5. Let $A$ be fuzzy set (FS) in $X=\left\{x_{1}, x_{2}, \ldots, x_{n}\right\}$, I and II be two subsets of the attribute set $X$, such that IUII $=X$, InII $=\phi$. If $x_{j} \in \mathrm{I}$, the value of fuzzy set $A$ is characterized by IFSs, if $x_{j} \in \mathrm{II}$, the values of fuzzy set $A$ is characterized by IVIFSs, then $A$ is called hybrid intuitionistic fuzzy sets (HIFSs).

Definition 6. Let $A=\left\{\left(x_{j}, \mu_{A}\left(x_{j}\right), v_{A}\left(x_{j}\right)\right) \mid x_{j} \in X\right\}$ and $B=$ $\left\{\left(x_{j}, \mu_{B}\left(x_{j}\right), v_{B}\left(x_{j}\right)\right) \mid x_{j} \in X\right\}$ be two hybrid intuitionistic fuzzy sets, such that if the same attributes $x_{j} \in \mathrm{I},\left(\mu_{A}\left(x_{j}\right)\right.$, $\left.v_{A}\left(x_{j}\right)\right)$, and $\left(\mu_{B}\left(x_{j}\right), v_{B}\left(x_{j}\right)\right)$ are IFSs, if the same attribute $x_{j} \in \mathrm{II}, \quad\left(\left[\mu_{A L}\left(x_{j}\right), \mu_{A U}\left(x_{j}\right)\right],\left[v_{A L}\left(x_{j}\right), v_{A U}\left(x_{j}\right)\right]\right), \quad$ and $\left(\left[\mu_{B L}\left(x_{j}\right), \mu_{B U}\left(x_{j}\right)\right],\left[v_{B L}\left(x_{j}\right), v_{B U}\left(x_{j}\right)\right]\right)$ are IVIFSs, which we call $A$ and $B$ the same type hybrid intuitionistic fuzzy sets.

Definition 7. Suppose $A$ and $B$ are the same type hybrid intuitionistic fuzzy sets, that is, if $x_{j} \in \mathrm{I},\left(\mu_{A}\left(x_{j}\right), v_{A}\left(x_{j}\right)\right)$, and $\left(\mu_{B}\left(x_{j}\right), v_{B}\left(x_{j}\right)\right)$ are IFSs, if the same attribute $x_{j} \in$ II, $\left(\left[\mu_{A L}\left(x_{j}\right), \mu_{A U}\left(x_{j}\right)\right],\left[v_{A L}\left(x_{j}\right), v_{A U}\left(x_{j}\right)\right]\right), \quad$ and $\left(\left[\mu_{B L}\left(x_{j}\right), \mu_{B U}\left(x_{j}\right)\right],\left[v_{B L}\left(x_{j}\right), v_{B U}\left(x_{j}\right)\right]\right)$ are IVIFSs, then the cosine similarity measure between hybrid intuitionistic fuzzy sets $A$ and $B$ is defined as follows:

$$
\begin{aligned}
C_{\mathrm{HIFS}}(A, B)= & \frac{1}{n}\left[\sum_{x_{i} \in \mathrm{I}} \frac{\mu_{A}\left(x_{i}\right) \mu_{B}\left(x_{i}\right)+v_{A}\left(x_{i}\right) \nu_{B}\left(x_{i}\right)+\pi_{A}\left(x_{i}\right) \pi_{B}\left(x_{i}\right)}{\sqrt{\mu_{A}^{2}\left(x_{i}\right)+v_{A}^{2}\left(x_{i}\right)+\pi_{A}^{2}\left(x_{i}\right)} \sqrt{\mu_{B}^{2}\left(x_{i}\right)+v_{B}^{2}\left(x_{i}\right)+\pi_{B}^{2}\left(x_{i}\right)}}\right. \\
& +\sum_{x_{i} \in \mathrm{II}} \frac{\mu_{A L}\left(x_{i}\right) \mu_{B L}\left(x_{i}\right)+\mu_{A U}\left(x_{i}\right) \mu_{B U}\left(x_{i}\right)+v_{A L}\left(x_{i}\right) v_{B L}\left(x_{i}\right)+v_{A U}\left(x_{i}\right) v_{B U}\left(x_{i}\right)+\pi_{A L}\left(x_{i}\right) \pi_{B L}\left(x_{i}\right)+\pi_{A U}\left(x_{i}\right) \pi_{B U}\left(x_{i}\right)}{\sqrt{\mu_{A L}^{2}\left(x_{i}\right)+\mu_{A U}^{2}\left(x_{i}\right)+v_{A L}^{2}\left(x_{i}\right)+v_{A U}^{2}\left(x_{i}\right)+\pi_{A L}^{2}\left(x_{i}\right)+\pi_{A U}^{2}\left(x_{i}\right)} \cdot \sqrt{\mu_{B L}^{2}\left(x_{i}\right)+\mu_{B U}^{2}\left(x_{i}\right)+v_{B L}^{2}\left(x_{i}\right)+v_{B U}^{2}\left(x_{i}\right)+\pi_{B L}^{2}\left(x_{i}\right)+\pi_{B U}^{2}\left(x_{i}\right)}} .
\end{aligned}
$$

Remark 2. If $\mathrm{I}=\phi$, then $C_{\mathrm{HIFS}}$ measure is reduced to $C_{\mathrm{IVIFS}}$ measure.
Remark 3. If II $=\phi$, then $C_{\mathrm{HIFS}}$ measure is reduced to $C_{\mathrm{IFS}}$ measure. 
Theorem 1. The cosine similarity measure between two hybrid intuitionistic fuzzy sets $A$ and $B$ satisfies the following properties:

(1) $0 \leq C_{\mathrm{HIFS}}(A, B) \leq 1$

(2) $C_{\mathrm{HIFS}}(A, B)=C_{\mathrm{HIFS}}(B, A)$

(3) $C_{\mathrm{HIFS}}(A, B)=1$ if $A=B$

Proof

(1) It is obvious that the property (1) is true according to the cosine value in $[0,1]$

(2) Because the multiplication of numbers satisfies the commutative law, if the positions of $A$ and $B$ are exchanged in the computation of cosine measure, the result values will not change, so the property (3) is true.

(3) If $A=B, x_{i} \in \mathrm{I}$, we have $\mu_{A}\left(x_{i}\right)=\mu_{B}\left(x_{i}\right)$ and $v_{A}\left(x_{i}\right)=v_{B}\left(x_{i}\right)$.

If $A=B, x_{i} \in \mathrm{II}$, we have $\mu_{A L}\left(x_{i}\right)=\mu_{B L}\left(x_{i}\right), \mu_{A U}\left(x_{i}\right)=$ $\mu_{B U}\left(x_{i}\right), v_{A L}\left(x_{i}\right)=v_{B L}\left(x_{i}\right)$, and $v_{A U}\left(x_{i}\right)=v_{B U}\left(x_{i}\right)$, then $C_{\text {HIFS }}(A, B)=1$ is obvious obtained.

\section{Multiple-Attribute Group Decision-Making with the Cosine Similarity Measure between Hybrid Intuitionstic Fuzzy Sets}

In this section, we will apply the $C_{\text {HIFS }}$ measure between hybrid intuitionistic fuzzy sets to medical diagnosis. The $C_{\text {HIFS }}$ measure can be applied in many situations, such as pattern recognition, medical diagnosis, and so on. The main motivation for considering this model is that the representation of the decision information is very complex. We need several doctors correctly to evaluate the symptoms of the disease. The doctor usually provides his/her preferences for symptoms with IFSs or IVIFSs. Suppose that doctors are good at different diagnostic skills, we can obtain the weights of doctors based on the projection of individual decision on the ideal decision; then, all individual diagnosis decisions are aggregated into a collective one. At last, we apply the $C_{\mathrm{HIFS}}$ measure to medical diagnosis.

In a given pathology, suppose that a set of symptoms $S=\left(s_{1}, s_{2}, \ldots, s_{n}\right)$, a set of diagnoses $A=\left(A_{1}, A_{2}, \ldots, A_{m}\right)$ and a set of medical experts $E=\left(e_{1}, e_{2}, \ldots, e_{t}\right)$. Assume that a patient has all the symptoms, which can be represented by the hybrid intuition fuzzy set $\widetilde{B}$, our aim is to diagnose what kind of diagnoses the patient $\widetilde{B}$ belong to.

In order to solve this problem, we first introduce some relevant concepts.

Definition 8. Let $A^{\prime}=\left(a_{i j}\right)_{m \times n}=\left(\mu_{A_{i}}\left(x_{j}\right), v_{A_{i}}\left(x_{j}\right)\right)_{m \times n}$ be a decision matrix, I and II be two subsets of the attribute set $X=\left\{x_{j} \mid j=1,2, \ldots, n\right)$, such that IUII $=X$ and InII $=\varnothing$. If the attribute $x_{j} \in \mathrm{I}$, then the evaluation values $a_{i j}$ are IFSs, if the attribute $x_{j} \in \mathrm{II}$, then the evaluation values $a_{i j}$ are IVIFSs. In this case, $A^{\prime}$ is called a hybrid intuitionistic fuzzy matrix.
Definition 9. Let $A^{\prime}=\left(A_{1}, A_{2}, \ldots, A_{m}\right)^{T}$ and $B^{\prime}=\left(B_{1}\right.$, $\left.B_{2}, \ldots, B_{m}\right)^{T}$ be two hybrid intuitionistic fuzzy matrices, where $A_{i}=\left(a_{i 1}, a_{i 2}, \ldots, a_{i n}\right)$ and $B_{i}=\left(b_{i 1}, b_{i 2}, \ldots, b_{i n}\right)(i=$ $1,2, \ldots m)$, if they satisfy the following conditions:

(1) $a_{i j}=\left(\mu_{A_{i}}\left(x_{j}\right), v_{A_{i}}\left(x_{j}\right)\right)$ about $x_{j}$ in $A_{i}$ if and only if $b_{i j}=\left(\mu_{B i}\left(x_{j}\right), v_{B i}\left(x_{j}\right)\right)$ about $x_{j}$ in $B_{i}$

(2) $a_{i}=\left(\left[\mu_{A_{i} L}\left(x_{j}\right), \mu_{A_{i} U}\left(x_{j}\right)\right],\left[\nu_{A_{i} L}\left(x_{j}\right), v_{A_{i} U}\left(x_{j}\right)\right]\right)$ about $x_{j}$ in $A_{i}$ if and only if $b_{i j}=\left(\left[\mu_{B_{i} L}\left(x_{j}\right), \mu_{B_{i} U}\left(x_{j}\right)\right]\right.$, $\left.\left[v_{B_{i} L}\left(x_{j}\right), v_{B_{i} U}\left(x_{j}\right)\right]\right)$ about $x_{j}$ in $B_{i}$, then $A_{i}$ and $B_{i}$ are the same type vector, $A^{\prime}$ and $B^{\prime}$ are the same type hybrid intuitionistic fuzzy matrices.

Now, suppose that a set of diagnoses $A^{\prime}=\left(A_{1}\right.$, $\left.A_{2}, \ldots, A_{m}\right)$, where $A_{i}$ is represented by IFS $A_{i}=\left(x_{j}, \mu_{A_{i}}\right.$ $\left.\left(x_{j}\right), v_{A_{i}}\left(x_{j}\right)\right)$ or IVIFS $A_{i}=\left(x_{j},\left[\mu_{A_{i} L}\left(x_{j}\right), \mu_{A_{i} U}\left(x_{j}\right)\right]\right.$, $\left.\left[v_{A_{i} L}\left(x_{j}\right), v_{A_{i} U}\left(x_{j}\right)\right]\right)(i=1,2, \ldots, m)$. We should diagnose what kind of disease the patient $\widetilde{B}$ belongs to. Furthermore, assume that the patient $\widetilde{B}$ is represented by the same type intuitionistic fuzzy set as $A_{i}$. In the following, we will present the method for application of $C_{\mathrm{HIFS}}$ measure to medical diagnosis, which involves the following steps:

Step 1. Each medical expert provides his/her individual decision matrix about the relation between the diagnosis and the symptoms.

Step 2. According to the expert's diagnostic decision matrix $R_{k}=\left(r_{i j}^{k}\right)_{m \times n}$, the ideal decision information should be close to the opinions of most doctors; then, we define the ideal relation $R^{*}=\left(r_{i j}^{*}\right)_{m \times n}$ between the diagnosis $A_{i}(i=1,2, \ldots$, $m)$ and the symptom $s_{j}(j=1,2, \ldots, n)$ as follows:

$$
\begin{aligned}
& R^{*}=\left(r_{i j}^{*}\right)_{m \times n}=\begin{array}{c}
A_{1} \\
A_{2} \\
\vdots \\
A_{m}
\end{array}\left(\begin{array}{cccc}
r_{11}^{*} & r_{12}^{*} & \cdots & r_{1 n}^{*} \\
r_{21}^{*} & r_{22}^{*} & \cdots & r_{2 n}^{*} \\
\vdots & \vdots & \cdots & \vdots \\
r_{m 1}^{*} & r_{m 2}^{*} & \cdots & r_{m n}^{*}
\end{array}\right) . \\
& \text { If } j \in \mathrm{I}, r_{i j}^{*}=\left(\mu_{i j}^{*}, v_{i j}^{*}\right)=\left(\frac{1}{t} \sum_{k=1}^{t} \mu_{i j}^{(k)}, \frac{1}{t} \sum_{k=1}^{t} v_{i j}^{(k)}\right) . \\
& \text { If } j \in I I, r_{i j}^{*}=\left(\tilde{\mu}_{i j}^{*}, \widetilde{v}_{i j}^{*}\right) \\
& =\left(\left[\frac{1}{t} \sum_{k=1}^{t} \mu_{i j L}^{(k)}, \frac{1}{t} \sum_{k=1}^{t} \mu_{i j U}^{(k)}\right],\left[\frac{1}{t} \sum_{k=1}^{t} v_{i j L}^{(k)}, \frac{1}{t} \sum_{k=1}^{t} v_{i j U}^{(k)}\right]\right) .
\end{aligned}
$$

Step 3. Medical experts may give unreasonable assessments when they encounter unfamiliar symptoms. So, it is not very reasonable to assume that each expert has equal weights. In order to obtain a reasonable evaluation, the weights of medical experts under different attributes are obtained by the projection of the individual evaluation on the ideal evaluation $r_{i j}^{*}$. The greater the weight of the expert is, the closer the evaluation value is to the ideal evaluation. 
The projection of each decision on the ideal decision is given by

$$
\begin{gathered}
\text { if } j \in \mathrm{I}, \operatorname{Pr} j_{r_{i j}^{*}}^{r_{i j}^{(k)}}=\frac{\mu_{i j}^{(k)} \mu_{i j}^{*}+v_{i j}^{(k)} v_{i j}^{*}+\pi_{i j}^{(k)} \pi_{i j}^{*}}{\sqrt{\mu_{i j}^{* 2}+v_{i j}^{* 2}+\pi_{i j}^{* 2}}}, \\
\text { if } j \in \mathrm{II}, \operatorname{Pr} j_{r_{i j}^{*}}^{r_{i j}^{(k)}}=\frac{\mu_{i j L}^{(k)} \mu_{i j L}^{*}+\mu_{i j U}^{(k)} \mu_{i j U}^{*}+v_{i j L}^{(k)} v_{i j L}^{*}+v_{i j U}^{(k)} v_{i j U}^{*}+\pi_{i j L}^{(k)} \pi_{i j L}^{*}+\pi_{i j U}^{(k)} \pi_{i j U}^{*}}{\sqrt{\mu_{i j L}^{* 2}+\mu_{i j U}^{* 2}+v_{i j L}^{* 2}+v_{i j U}^{* 2}+\pi_{i j L}^{* 2}+\pi_{i j U}^{* 2}}} .
\end{gathered}
$$

Then the weight of medical expert's evaluation on different symptoms can be defined as

$$
\begin{gathered}
w_{i j}^{(k)}=\frac{\operatorname{Pr} j_{r_{i j}^{*}} r_{i j}^{(k)}}{\sum_{k=1}^{t} \operatorname{Pr} j_{r_{i j}^{*}} r_{i j}^{(k)}}, \quad k=1,2, \ldots, t ; \\
i=1,2, \ldots, m ; j=1,2, \ldots, n .
\end{gathered}
$$

Step 4. According to the recognition principle of maximum degree of cosine similarity measure, the process of diagnosis $\widetilde{B}$ to $A_{k}$ is derived by $k=\operatorname{Max}_{1 \leq i \leq m}\left(C_{\mathrm{HIFS}}\left(A_{i}, \widetilde{B}\right)\right)$.

\section{Numerical Example}

In this section, the proposed cosine similarity measure between hybrid IFSs is applied in medical diagnosis to demonstrate its effectiveness.

5.1. Illustration of the Cosine Similarity Measures for Hybrid IFSs. Assume that a set of diagnosis $A=\left\{A_{1}\right.$ (viralfever), $A_{2}$ (typhoid), $A_{3}$ (stomach problem), $A_{4}$ (chest problem) $\}$ and a set of symptoms $S=\left\{s_{1}\right.$ (temperature), $s_{2}$ (stomach pain), $s_{3}$ (cough), $s_{4}$ (chest pain) $\}$. Suppose a patient has all the symptoms, which can be represented by the following hybrid intuitionistic fuzzy information (data obtained through a survey of doctors):

$$
\begin{aligned}
\widetilde{B}= & \left\{\left(s_{1}, 0.5,0.4\right),\left(s_{2}, 0.6,0.2\right),\left(s_{3},[0.5,0.6],[0.2,0.3]\right),\right. \\
& \left.\left(s_{4}, 0.4,0.2\right)\right\} .
\end{aligned}
$$

There are three medical experts evaluate each diagnosis with all the symptoms, which are represented by the hybrid IFSs, the results are shown in Tables 1-3.

By step 3 in Section 4, applying (12)-(14), we can calculate the weights of each medical expert for the diagnosis with respect to different symptoms, which are obtained in Tables 5-7.

From the previous formula $C_{\mathrm{HIFS}}\left(A^{\prime}, B\right)$, we can calculate the cosine similarity measure between $A_{i}(i=1,2,3,4)$ and $\widetilde{B}$ as follows:

$$
\begin{aligned}
C_{\mathrm{HIFS}}\left(\widetilde{A}_{1}, \widetilde{B}\right) & =0.9674, \\
C_{\text {HIFS }}\left(\widetilde{A}_{2}, \widetilde{B}\right) & =0.9477, \\
C_{\mathrm{HIFS}}\left(\widetilde{A}_{3}, \widetilde{B}\right) & =0.9140, \\
C_{\text {HIFS }}\left(\widetilde{A}_{4}, \widetilde{B}\right) & =0.9511 .
\end{aligned}
$$

We can conclude that the diagnosis of the patient $\widetilde{B}$ is viral fever $\left(A_{1}\right)$.

5.2. Comparison Analysis. In this subsection, the existing cosine similarity measure is used to compare with the same numerical example. In the numerical example, the decision information is represented with hybrid IFS, we can transform it into a unified form. For example, the relation between the diagnosis and the symptoms under the attribute $s_{3}$ of experts is IVIFSs, and if we use the cosine similarity measure $C_{\text {IFS }}$ proposed by Ye [7] to calculate the numerical example, we should convert the corresponding IVIFSs to IFS according to the midpoints of IVIFSs. For example, $([0.4,0.6],[0.1,0.3])$ can be converted to (0.5). Then using the cosine similarity measure $C_{\mathrm{IFS}}$ proposed by Ye [7], we can obtain the corresponding cosine similarity measure values: $C_{\mathrm{IFS}}\left(A_{1}, \widetilde{B}\right)=$ $0.9691, \quad C_{\mathrm{IFS}}\left(A_{2}, \widetilde{B}\right)=0.9546, \quad C_{\mathrm{IFS}}\left(A_{3}, \widetilde{B}\right)=0.9377$, and $C_{I F S}\left(A_{4}, \widetilde{B}\right)=0.9586$. That is to say, the diagnosis of the patient $\widetilde{B}$ is still the viral fever $A_{1}$. The proposed cosine similarity between hybrid IFS in this paper produces the same results as the existing methods. This means that the proposed method is feasible and effective, and it has some advantages in solving multiple criteria decision-making problems. On one hand, the method is more convenient to make decision for decision makers, who can express their preferences over the decision information by IFS or IVIFS simultaneously. On the other hand, because the information conversion will be lost in decision-making process, there are no information conversions between IFSs and IVIFSs in this model, the alternatives will be ranked directly based on the original decision information.

\section{Conclusion}

The paper proposed the cosine similarity measure between hybrid intuitionistic fuzzy sets, and the proposed method 
TABLE 1: The relation between the diagnosis and the symptoms-expert 1 .

\begin{tabular}{lcccc}
\hline & $s_{1}$ & $s_{2}$ & $s_{3}$ & $s_{4}$ \\
\hline$A_{1}$ & $(0.5,0.4)$ & $(0.5,0.3)$ & $([0.4,0.6],[0.1,0.3])$ & $(0.4,0.4)$ \\
$A_{2}$ & $(0.7,0.3)$ & $(0.7,0.2)$ & $([0.3,0.5],[0.4,0.5])$ & $(0.6,0.2)$ \\
$A_{3}$ & $(0.8,0.1)$ & $(0.6,0.4)$ & $([0.6,0.7],[0.2,0.3])$ & $(0.6,0.3)$ \\
$A_{4}$ & $(0.7,0.2)$ & $(0.5,0.2)$ & $([0.5,0.7],[0.1,0.2])$ & $(0.5,0.3)$ \\
\hline
\end{tabular}

TABLE 2: The relation between the diagnosis and the symptoms-expert 2.

\begin{tabular}{lcccc}
\hline & $s_{1}$ & $s_{2}$ & $s_{3}$ & $s_{4}$ \\
\hline$A_{1}$ & $(0.4,0.5)$ & $(0.6,0.2)$ & $([0.5,0.6],[0.2,0.3])$ & $(0.3,0.4)$ \\
$A_{2}$ & $(0.5,0.2)$ & $(0.7,0.2)$ & $([0.4,0.7],[0.1,0.3])$ & $(0.7,0.1)$ \\
$A_{3}$ & $(0.6,0.2)$ & $(0.5,0.1)$ & $([0.5,0.7],[0.1,0.2])$ & $(0.6,0.2)$ \\
$A_{4}$ & $(0.7,0.1)$ & $(0.4,0.3)$ & $([0.3,0.6],[0.2,0.4])$ & $(0.4,0.3)$ \\
\hline
\end{tabular}

TABLE 3: The relation between the diagnosis and the symptoms-expert 3.

\begin{tabular}{lcccc}
\hline & $s_{1}$ & $s_{2}$ & $s_{3}$ & $s_{4}$ \\
\hline$A_{1}$ & $(0.5,0.3)$ & $(0.6,0.2)$ & $([0.4,0.6],[0.2,0.3])$ & $(0.5,0.4)$ \\
$A_{2}$ & $(0.7,0.2)$ & $(0.4,0.4)$ & $([0.5,0.7],[0.1,0.3])$ & $(0.6,0.3)$ \\
$A_{3}$ & $(0.6,0.3)$ & $(0.7,0.3)$ & $([0.6,0.8],[0.1,0.2])$ & $(0.7,0.2)$ \\
$A_{4}$ & $(0.5,0.2)$ & $(0.5,0.3)$ & $([0.3,0.6],[0.1,0.4])$ & $(0.6,0.1)$ \\
\hline
\end{tabular}

According to step 2 in Section 4, applying (10) and (11), respectively, the ideal relation between the diagnosis and the symptoms are shown in Table 4 .

TABLE 4: The ideal relation between the diagnosis and the symptoms.

\begin{tabular}{ccccc}
\hline & $s_{1}$ & $s_{2}$ & $s_{3}$ & $s_{4}$ \\
\hline$A_{1}^{*}$ & $(0.467,0.4)$ & $(0.567,0.233)$ & $([0.433,0.6],[0.167,0.3])$ & $(0.4,0.4)$ \\
$A_{2}^{*}$ & $(0.633,0.233)$ & $(0.6,0.267)$ & $([0.4,0.633],[0.2,0.367])$ & $(0.633,0.2)$ \\
$A_{3}^{*}$ & $(0.667,0.2)$ & $(0.6,0.267)$ & $([0.567,0.733],[0.133,0.233])$ & $(0.633,0.233)$ \\
$A_{4}^{*}$ & $(0.633,0.167)$ & $(0.467,0.267)$ & $([0.367,0.633],[0.133,0.333])$ & $(0.5,0.233)$ \\
\hline
\end{tabular}

TABLE 5: The weights of the medical expert 1 for $A_{i}$ with respect to $s_{j}$.

\begin{tabular}{lcccc}
\hline & $s_{1}$ & $s_{2}$ & $s_{3}$ & $s_{4}$ \\
\hline$A_{1}$ & 0.3427 & 0.3155 & 0.3391 & 0.3333 \\
$A_{2}$ & 0.3614 & 0.3614 & 0.3052 & 0.3223 \\
$A_{3}$ & 0.3761 & 0.3466 & 0.3293 & 0.3263 \\
$A_{4}$ & 0.3458 & 0.3395 & 0.3314 & 0.3313 \\
\hline
\end{tabular}

TABLE 6: The weights of the medical expert 2 for $A_{i}$ with respect to $s_{j}$.

\begin{tabular}{lcccc}
\hline & $s_{1}$ & $s_{2}$ & $s_{3}$ & $s_{4}$ \\
\hline$A_{1}$ & 0.3371 & 0.3423 & 0.3311 & 0.3148 \\
$A_{2}$ & 0.2841 & 0.3614 & 0.3474 & 0.3531 \\
$A_{3}$ & 0.3097 & 0.2821 & 0.3196 & 0.3193 \\
$A_{4}$ & 0.3594 & 0.3210 & 0.3276 & 0.3106 \\
\hline
\end{tabular}

TABle 7: The weights of the medical expert 3 for $A_{i}$ with respect to $s_{j}$.

\begin{tabular}{lcccr}
\hline & $s_{1}$ & $s_{2}$ & $s_{3}$ & $s_{4}$ \\
\hline$A_{1}$ & 0.3202 & 0.3422 & 0.3298 & 0.3519 \\
$A_{2}$ & 0.3545 & 0.2772 & 0.3474 & 0.3246 \\
$A_{3}$ & 0.3142 & 0.3713 & 0.3511 & 0.3544 \\
$A_{4}$ & 0.2948 & 0.3395 & 0.3410 & 0.3581 \\
\hline
\end{tabular}

When the weight values of the experts are determined, the aggregated evaluating decision results provided by different experts are obtained in Table 8 . 
TABLE 8: The aggregated relation between the diagnosis and the symptom.

\begin{tabular}{lcccc}
\hline & $s_{1}$ & $s_{2}$ & $s_{3}$ & $s_{4}$ \\
\hline$A_{1}$ & $(0.4683,0.3933)$ & $(0.5708,0.2273)$ & $([0.4351,0.6],[0.1581,0.3])$ & $(0.4093,0.4)$ \\
$A_{2}$ & $(0.5316,0.2316)$ & $(0.6364,0.2424)$ & $([0.4097,0.6494],[0.1527,0.3506])$ & $(0.6386,0.1786)$ \\
$A_{3}$ & $(0.6918,0.175)$ & $(0.6172,0.2431)$ & $([0.5704,0.7398],[0.1256,0.2286])$ & $(0.6388,0.2283)$ \\
$A_{4}$ & $(0.6513,0.1559)$ & $(0.4699,0.2614)$ & $([0.3739,0.6364],[0.1255,0.3179])$ & $(0.5115,0.2024)$ \\
\hline
\end{tabular}

would be quite good for some real-world applications, such as pattern recognition and medical diagnosis. Through the proposed cosine similarity measure, we can classify the patient $\widetilde{B}$ in one of the diagnosis $A_{1}, A_{2}, \ldots A_{m}$. Finally, a numerical example illustrated the application and efficiency of the developed approach, which is also compared to the existing methods. In future research, we expect to develop further extensions of the $C_{\text {HIFS }}$ measure by adding the new characteristic, such as ordered weighted averaging operator, and we will also consider other applications of the proposed $C_{\mathrm{HIFS}}$ measure.

\section{Abbreviations}

IFS: Intuitionistic fuzzy set

IVIFS: Interval-valued intuitionistic fuzzy set

HIFS: Hybrid intuitionistic fuzzy set

$C_{\mathrm{IFS}}$ : Cosine similarity measure for intuitionistic fuzzy set

$C_{\text {IVIFS }}$ : Cosine similarity measure for interval-valued intuitionistic fuzzy set

$C_{\mathrm{HIFS}}$ : Cosine similarity measure for hybrid intuitionistic fuzzy set.

\section{Data Availability}

No data were used to support this study.

\section{Conflicts of Interest}

The authors declare that they have no conflicts of interest regarding the publication for the paper.

\section{Acknowledgments}

This research was fully supported by the Youth Project of Hunan Education Department (17B092), the Key Project of National Nature Science Foundation of China (Nos. 71431006 and 11501191), a grant from the National Natural Science Foundation of Hunan (2017JJ2096), the National Social Science Fund of China (15BTJ028), and the Major Projects of the National Social Science Foundation of China (17ZDA046). We thank the editor and anonymous reviewers for their helpful comments on an earlier draft of this paper.
[3] D. Li and C. Cheng, "New similarity measures of intuitionistic fuzzy sets and application to pattern recognitions," Pattern Recognition Letters, vol. 23, no. 1-3, pp. 221-225, 2002.

[4] Z. Liang and P. Shi, "Similarity measures on intuitionistic fuzzy sets," Pattern Recognition Letters, vol. 24, no. 15, pp. 2687-2693, 2003.

[5] D. Liu, X. Chen, and D. Peng, "The intuitionistic fuzzy linguistic cosine similarity measure and its application in pattern recognition," Complexity, vol. 2018, Article ID 9073597, 11 pages, 2018.

[6] C. P. Pappis and N. I. Karacapilidis, "A comparative assessment of measures of similarity of fuzzy values," Fuzzy Sets and Systems, vol. 56, no. 2, pp. 171-174, 1993.

[7] J. Ye, "Cosine similarity measures for intuitionistic fuzzy sets and their applications," Mathematical and Computer Modelling, vol. 53, no. 1-2, pp. 91-97, 2011.

[8] L. Zhou, Z. Tao, H. Chen et al., "Intuitionistic fuzzy ordered weighted cosine similarity measure," Group Decision and Negotiation, vol. 23, no. 4, pp. 879-900, 2014.

[9] H. Nguyen, "A novel similarity/dissimilarity measure for intuitionistic fuzzy sets and its application in pattern recognition," Expert Systems with Applications, vol. 45, pp. 97-107, 2016.

[10] K. T. Atanassov and G. Gargov, "Interval valued intuitionistic fuzzy sets intuitionistic fuzzy sets," Fuzzy Sets and Systems, vol. 31, no. 3, pp. 343-349, 1989.

[11] Z. Xu, "On similarity measures of interval-valued intuitionistic fuzzy sets and their application to pattern recognitions," Journal of Southeast University, vol. 23, no. 1, 2007.

[12] A. Bhattacharyya, "On a measure of divergence between two multinomial populations," Sankhya, vol. 7, no. 4, pp. 401-406, 1946.

[13] J. Ye, "Interval-valued intuitionistic fuzzy cosine similarity measures for multiple attribute decision-making," International Journal of General Systems, vol. 42, no. 8, pp. 883-891, 2013.

[14] Y. L. Zhou and M. A. Wahab, "Cosine based and extended transmissibility damage indicators for structural damage detection," Engineering Structures, vol. 141, pp. 175-183, 2017.

[15] Y. L. Zhou, N. M. Maia, and M. A. Wahab, "Damage detection using transmissibility compressed by principal component analysis enhanced with distance measure," Journal of $\mathrm{Vi}$ bration and Control, vol. 24, no. 10, 2017.

[16] K.T. Atanassov, "Intuitionistic fuzzy sets," Fuzzy Sets and Systems, vol. 20, no. 1, pp. 87-96, 1986.

[17] Z.S. Xu and J. Chen, "On geometric aggregation over intervalvalued intuitionistic fuzzy information," in Proceedings of International Conference on Fuzzy Systems and Knowledge Discovery, pp. 466-471, IEEE, Haikou, Hainan, China, 2007.

\section{References}

[1] S. M. Chen, "Measures of similarity between vague sets," Fuzzy Sets and Systems, vol. 74, no. 2, pp. 217-223, 1995.

[2] W. L. Hung and M. S. Yang, "Similarity measures of intuitionistic fuzzy sets based on Hausdorff distance," Pattern Recognition Letters, vol. 25, no. 14, pp. 1603-1611, 2004. 


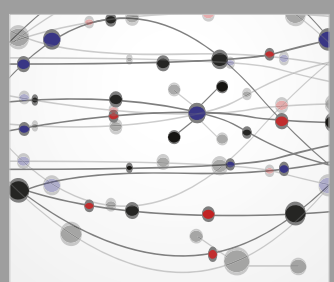

The Scientific World Journal
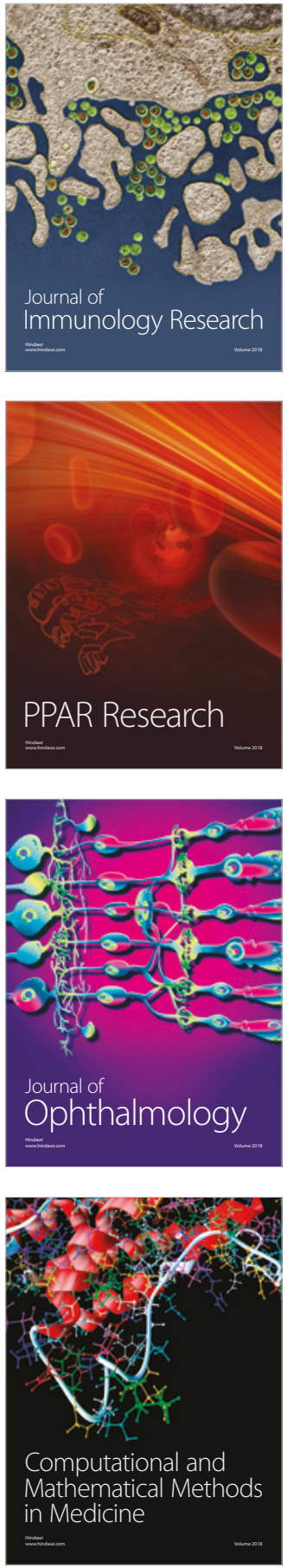

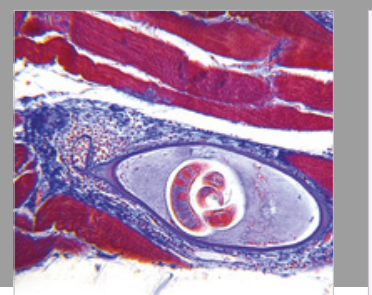

Gastroenterology Research and Practice

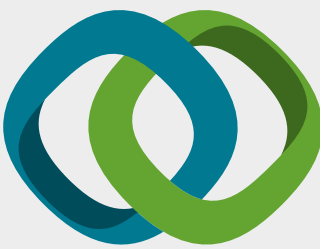

\section{Hindawi}

Submit your manuscripts at

www.hindawi.com
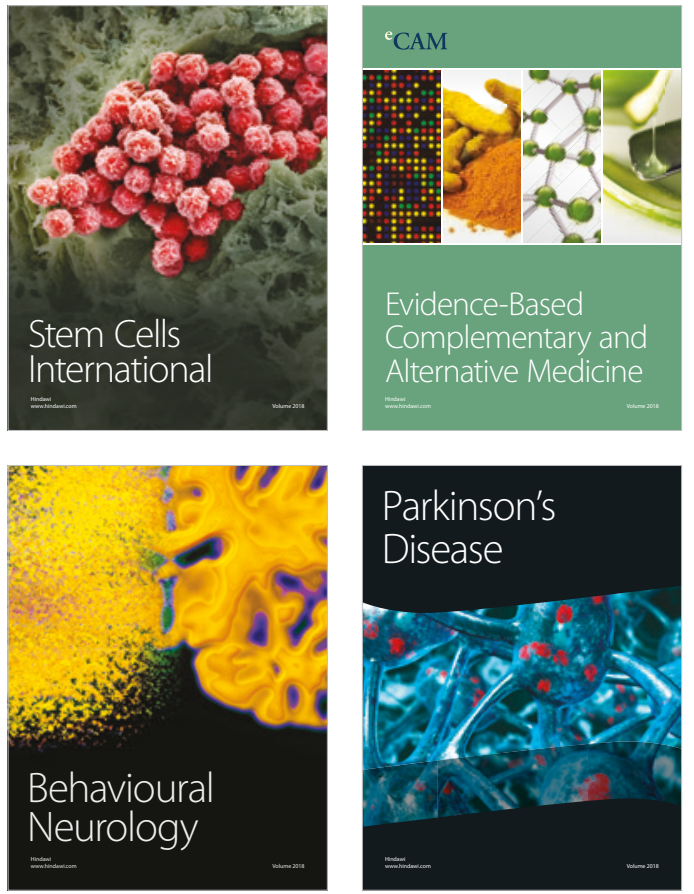

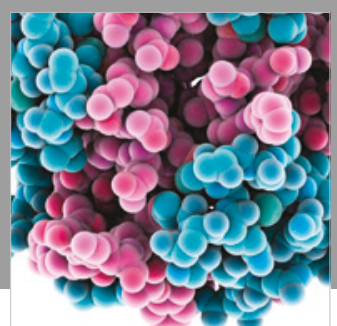

ournal of

Diabetes Research

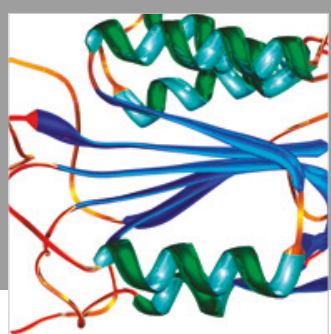

Disease Markers
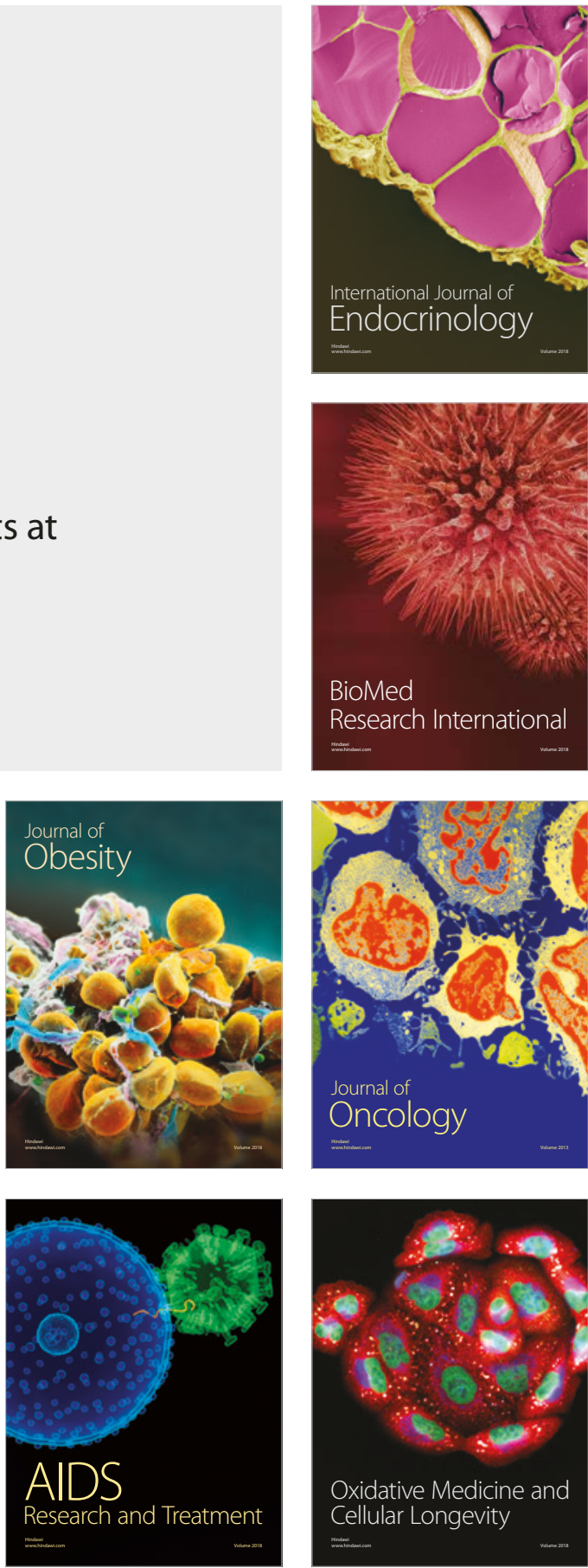